Article

\title{
Comparison of [68Ga]-FAPI PET/CT and [18F]-FDG PET/CT in Multiple Myeloma: Clinical Experience
}

\author{
Umut Elboga ${ }^{1, *}$, Ertan Sahin ${ }^{1}$, Yusuf Burak Cayirli ${ }^{1}$, Merve Okuyan ${ }^{1}$, Gokmen Aktas ${ }^{2}$, \\ Handan Haydaroglu Sahin ${ }^{3}$, Ilkay Dogan ${ }^{4}{ }^{1}$, Tulay Kus ${ }^{5}$, Dervis Murat Akkurd ${ }^{3}$, Ufuk Cimen ${ }^{1}$, Vuslat Mumcu ${ }^{1}$, \\ Benan Kilbas ${ }^{6}$ and Yusuf Zeki Celen ${ }^{1}$
}

check for

updates

Citation: Elboga, U.; Sahin, E.;

Cayirli, Y.B.; Okuyan, M.; Aktas, G.; Haydaroglu Sahin, H.; Dogan, I.; Kus, T.; Akkurd, D.M.; Cimen, U.; et al. Comparison of [68Ga]-FAPI PET/CT and [18F]-FDG PET/CT in Multiple Myeloma: Clinical Experience.

Tomography 2022, 8, 293-302. https:// doi.org/10.3390/tomography8010024

Academic Editor: Emilio Quaia

Received: 29 December 2021

Accepted: 26 January 2022

Published: 1 February 2022

Publisher's Note: MDPI stays neutral with regard to jurisdictional claims in published maps and institutional affiliations.

Copyright: (C) 2022 by the authors. Licensee MDPI, Basel, Switzerland. This article is an open access article distributed under the terms and conditions of the Creative Commons Attribution (CC BY) license (https:// creativecommons.org/licenses/by/ $4.0 /)$.
1 Department of Nuclear Medicine, Gaziantep University, Gaziantep 27310, Turkey; ertansah@gantep.edu.tr (E.S.); yusufburakcayirli@gantep.edu.tr (Y.B.C.); merveokuyan@gantep.edu.tr (M.O.); ufuk.cimen1992@hotmail.com (U.C.); vuslatmumcu@hotmail.com (V.M.); celen@gantep.edu.tr (Y.Z.C.)

2 Department of Oncology, Medical Park Private Hospital, Gaziantep 27090, Turkey; gokmen.aktas@medicalpark.com.tr

3 Department of Hematology, Gaziantep University, Gaziantep 27310, Turkey; handansahin@gantep.edu.tr (H.H.S.); dervismuratakkurd@gantep.edu.tr (D.M.A.)

4 Department of Biostatistics, Gaziantep University, Gaziantep 27310, Turkey; ilkaydogan@gantep.edu.tr

5 Department of Oncology, Gaziantep University, Gaziantep 27310, Turkey; tulaykus@gantep.edu.tr

6 Department of R\&D, Moltek Health Services Production \& Marketing Inc., Kocaeli 41400, Turkey; benan.kilbas@moltek.com.tr

* Correspondence: umutelboga@gantep.edu.tr

\begin{abstract}
Objective: In this study, we aimed to compare [68Ga]FAPI PET/CT and [18F]FDG PET/CT imaging to detect lesions in multiple myeloma. Methods: A total of 14 patients with multiple myeloma who underwent [68Ga]FAPI PET/CT and [18F]FDG PET/CT imaging were included in this retrospective study. SUV $\max$ values of [68Ga]FAPI and [18F]FDG were compared according to lesion locations. Also, lesion localization ability of both imaging methods was compared on the patient basis. Results: In 4 of 14 patients, [68Ga]FAPI PET/CT and [18F]FDG PET/CT have not detected any bone lesions. In 8 of the remaining 10 patients [18F]FDG PET/CT detected bone lesions but in this group, 6 patients showed more higher $\mathrm{SUV}_{\max }$ values than [18F]FDG PET/CT in [68Ga]FAPI PET/CT.In contrast, 2 of 8 patients showed more higher $\mathrm{SUV}_{\max }$ values than [68Ga]FAPI PET/CT in [18F]FDG PET/CT. Moreover, [68Ga]FAPI PET/CT detected bone lesions in two patients, which werenot detected by [18F]FDG PET/CT. Also, in five patients, [68Ga]FAPI PET/CT showed more bone lesions in comparison with[18F]FDG PET/CT. Only one patient, [18F]FDG PET/CT showed more bone lesions. Three extramedullary involvements were observed in the following locations: lung, presacral lymph node, and soft tissue mass lateral to the right maxillary sinus. Among these involvements, higher $S_{U V} V_{\max }$ values were observed in the lung and presacral lymph node with [68Ga]FAPI compared to [18F]FDG. However, the soft tissue mass showed a higher $\mathrm{SUV}_{\max }$ value in [18F]FDG than [68Ga]FAPI. Conclusions: No significant superiority was observed in [68Ga]FAPI PET/CT over [18F]FDG PET/CT in patients with MM. However, [68Ga]FAPI PET/CT can be utilized as a complementary imaging method to [18F]FDG PET/CT in some settings, especially in low[18F]FDG affinity and inconclusive cases. Considering the favorable aspects of [68Ga]FAPI PET/CT in MM, such as low background activity, absence of non-specific bone marrow, and physiological brain involvement, further studies with a larger sample size should be conducted.
\end{abstract}

Keywords: multiple myeloma; [68Ga]FAPI; [18F]FDG

\section{Introduction}

Multiple myeloma (MM) is a neoplastic disease of the bone characterized by uncontrolled clonal proliferation of plasma cells. Bone disease, one of the major causes of mortality and morbidity in MM, occurs at the time of diagnosis in approximately two-thirds 
of patients and during disease in almost all patients [1,2]. For this reason, imaging plays a very important role in the diagnosis of $\mathrm{MM}$, which ismainly based on bone involvement. First, detection of osteolytic bone lesions and end-organ damage closely associated with the disease is essential to determine the need for immediate treatment [3]. Unlike other malignancies that metastasize to the bone, bone lesions in MM do not cause new bone formation as they are lytic in nature [4]. Skeletal lesions are seen in the spine, pelvis, skull, ribs, sternum, and proximal appendicular skeleton, in order of frequency [5]. Skeletal examinations have been replaced by whole body CT (computed tomography), whole body MRI (magnetic resonance imaging), or [18F]FDG PET/CT (18F-fluoro-deoxy-glucose positron emission tomography) in routine studies to identify lesions that define MM. Both MRI and [18F]FDG PET/CT have the advantage of evaluating bone marrow-occupying lesions before bone resorption is seen on CT [6]. The European Myeloma Network and European Society for Medical Oncology guidelines have recommended whole body CT as the imaging modality of choice for the initial assessment of MM-related lytic bone lesions and MRI is the gold standard imaging modality for detecting bone marrow involvement. However, [18F]FDG PET/CT provides valuable prognostic data and is preferred for evaluating response to treatment. Previous studies have shown that [18F]FDG PET/CT aid is useful in detecting both osseous and extra osseous MM related lesions [7-9]. On the other hand, low hexokinase-2 expression in MM may cause false negative results [10]. Furthermore, recent steroid treatment, small lytic lesions in the skull close to the brain, and hyperglycemia are other possible causes of false negative results on [18F]FDG PET/CT [11]. Although [18F]FDG-avid lesions and extramedullary involvement of MM are associated with a poor prognosis, possible false negative results, as mentioned above, may have a negative clinical impact on the initial assessment of MM [12].

Fibroblast activation protein (FAP), a member of the dipeptidyl peptidase IV (DPP-IV) family, is expressed on the surface of cancer-associated fibroblasts (CAF). Therefore, FAP expression is subjected to both diagnostic and therapeutic studies $[13,14]$. Studies on FAP have drawn attention to increased expression in various cancers. Although FAP expression is high in cancer stroma, it is considerably low in normal adult tissues, except for sites of active tissue damage, chronic inflammation, and remodeling [15]. The relatively specific expression of FAP in the tumor microenvironment has made it possible to develop FAP inhibitors (FAPIs) [16,17]. Subsequently, [68Ga]-labeled FAPI provided PET/CT images with high tumor-to-background ratios (TBRs) in a wide variety of cancer patients, suggesting high potential for FAP-targeted diagnosis and possibly targeted, radioligand therapies in the future. Considering that [68Ga]FAPI PET/CT demonstrates low background activity, including bones, it may be beneficial in MM lesions with low [18F]FDG affinity $[18,19]$.

In this study, we aim to compare [68Ga]FAPI PET/CT and [18F]FDG PET/CT in terms of bone or extramedullary involvement in multiple myeloma.

\section{Materials and Methods}

\subsection{Patients}

This retrospective study was approved by the Clinical Research Ethics Committee of our university and conducted in accordance with the 1964 Helsinki declaration for ethical standards. For cases with low [18F]FDG affinity, [68Ga]FAPI PET/CT was applied after [18F]FDG PET/CT enrolled in this study between September 2020 and February 2021, within the scope of the local institutional license for the magistral production and use of [68Ga]-labeled radiopharmaceuticals, and the use of experimental radiopharmaceuticals in determined patient groups retrospectively scanned from the medical record archive. Inclusion criteria were (1) being older than 18 years, (2) having histopathological confirmation of MM, and (3) being able to provide informed consent. Patients' exclusion criteria were (1) pregnancy or lactation; (2) inability or unwillingness to provide written informed consent;and (3) having arthritis, chronic inflammatory condition, or cirrhosis. Furthermore, levels of LDH (lactate dehydrogenase), CRP (C-reactive protein), and beta-2 microglobulin 
were obtained alongside histopathological features and immunoglobulin secretion situation in all patients.

\subsection{Staging in Multiple Myeloma: International Staging System (ISS) and Durie and Salmon PLUS Staging System}

Beta-2-microglobulin levels are divided into three stages according to ISS criteria [20-22]. Accordingly, patients with $\beta_{2}$-microglobulin levels less than $3.5 \mathrm{mg} / \mathrm{L}$ were classified as stage I, patients with serum $\beta_{2}$-microglobulin levels equal to or higher than $3.5 \mathrm{mg} / \mathrm{L}$ and less than $5.5 \mathrm{mg} / \mathrm{L}$ were classified as stage II, and patients with a serum $\beta_{2}$-microglobulin level equal to or higher than $5.5 \mathrm{mg} / \mathrm{L}$ were stage III [22].

The Salmon-Durie classification of MM is based on three stages and additional subclassifications. In stage I, the MM cell mass is less than $0.6 \times 10^{12}$ cells $/ \mathrm{m}^{2}$, and all the following are present: hemoglobin value $>10 \mathrm{~g} / \mathrm{dL}$, serum calcium value $<12 \mathrm{mg} / \mathrm{dL}$ (normal), normal bone structure (scale 0 ) or only a solitary bone plasmacytoma on radiographs, low M-component production rates (IgG value $<5 \mathrm{~g} / \mathrm{dL}$, IgA value $<3 \mathrm{~g} / \mathrm{dL}$, urine light-chain $\mathrm{M}$ component on electrophoresis $<4 \mathrm{~g} / 24 \mathrm{~h})$. In stage II, the MM cell mass is 0.6-1.2 $\times 10^{12}$ cells $/ \mathrm{m}^{2}$ or more. The other values fit neither those of stage I nor those of stage III. In stage III, the MM cell mass is $>1.2 \times 10^{12}$ cells $/ \mathrm{m}^{2}$, and all of the following are present: hemoglobin value $<8.5 \mathrm{~g} / \mathrm{dL}$, serum calcium value $>12 \mathrm{mg} / \mathrm{dL}$, advanced lytic bone lesions (scale 3) on radiographs, high $\mathrm{M}$-component production rates (IgG value greater than $7 \mathrm{~g} / \mathrm{dL}$, IgA value greater than $5 \mathrm{~g} / \mathrm{dL}$, urine light-chain M component on electrophoresis greater than $12 \mathrm{~g} / 24 \mathrm{~h}$ ). Integration of imaging the MRI or PET findings were used to stage the disease in each patient according to the Durie and Salmon PLUS staging system: stage I (0-4 lesions), stage II (5-20 lesions), and stage III (>20 lesions) [23-25].

\section{3. [68. Ga]FAPI-04}

FAPI-04 was obtained from MedChem Express LLC. The pharmaceutical grade $(68 \mathrm{Ge}) /(68 \mathrm{Ga})$ generator $(50 \mathrm{mCi})$ and disposable cassettes were supplied by Eckert and Ziegler Eurotope GmbH. Purification cartridge CM (Sep-Pak AccellPlus CM Plus Light Cartridge, 130 mg Sorbent per Cartridge, 37-55 $\mu \mathrm{m}$, WAT023531) was well-established in the cassette accessories. Other chemicals and materials were purchased from Aldrich (Chemical Company Inc., Istanbul, Turkey) in ultra-pure and trace metal basis grades. The HPLC analyses were performed by Modular-Lab HPLC (Eckert and Ziegler Inc., Wilmington, DC, USA) device using ACE-3 C18 $150 \times 3.0 \mathrm{~mm}^{2}$ column.

\subsection{Radiolabeling Procedure}

The radiolabeling process was performed by a fully automated system without any manual interaction. $(68 \mathrm{Ga})^{3+}$ was eluted with $0.1 \mathrm{~N} \mathrm{HCl}$ solution $(8.0 \mathrm{~mL})$ followed by passing through the pre-concentration on a strong cation exchange (SCX) cartridge. The (68Ga) activity was recovered from the SCX cartridge by $0.9 \mathrm{~mL}$ eluent $(5 \mathrm{M} \mathrm{NaCl} / \mathrm{HCl}$ $(0.1 \mathrm{M}))$. The reaction vial is filled by $2 \mathrm{~mL}$ of $\mathrm{H}^{2} \mathrm{O}, 0.4 \mathrm{~mL}$ of sodium acetate buffer $(\mathrm{pH}$ is around 4.5$), 0.2 \mathrm{~mL}$ of ethanol, and $50 \mu \mathrm{g}$ of FAPI-04. Then, (68Ga) activity was transferred to the reaction vial, and it was heated to $95{ }^{\circ} \mathrm{C}$ for $10 \mathrm{~min}$. After the completion of the reaction, the reaction medium was cooled down and crude product was diluted by adding $5.0 \mathrm{~mL}$ of $0.9 \% \mathrm{NaCl}$ and subsequently purified by the CM cartridge. Finally, the reaction mixture was passed through a millipore filter $(0.22 \mu \mathrm{m})$ and was injected intravenously after more than a $98 \%$ radiochemical purity with $88 \%$ radiochemical yield. The radiochemical purity was analyzed by R-HPLC and free $(68 \mathrm{Ga})$ was detected at RT $=2.2 \mathrm{~min}$, whereas [68Ga]FAPI-04 was detected at RT $=3.99 \mathrm{~min}$. (ACE-3 C18 $150 \times 3.0 \mathrm{~mm}^{2}$ column, isocratic flow $0.6 \mathrm{~mL} / \mathrm{min}$; mobile phase: $85 \% \mathrm{H}^{2} \mathrm{O}(0.1 \mathrm{TFA})$ and $15 \% \mathrm{ACN}(0.1 \mathrm{TFA})$.

\subsection{PET/CT Protocol and Image Analysis}

All patients were examined using a PET/CT system (Discovery ${ }^{\mathrm{TM}}$ IQ; GE Healthcare, Milwaukee, Brookfield, WI, USA) combining a dedicated, five-ring PET scanner with 
Light Burst Technology Inc. (London, UK). All patients had fasted for at least $6 \mathrm{~h}$ before [18F]FDG administration. Blood glucose was tested to ensure a normal blood glucose level. A whole-body (from top of head to mid-thigh) PET/CT was performed approximately $60 \mathrm{~min}$ after the intravenous injection of [18F]FDG $(3.7-5.4 \mathrm{MBq} / \mathrm{kg}, 0.10-0.15 \mathrm{mCi} / \mathrm{kg})$ according to the clinical standard protocol for tumor imaging. All patients underwent whole-body [68Ga]FAPI PET/CT within one week, with no specific preparation required before [68Ga]FAPI administration. The PET/CT scan was performed after the intravenous injection of [68Ga]FAPI (1.85-3.7 MBq $/ \mathrm{kg}, 0.05-0.1 \mathrm{mCi} / \mathrm{kg})$.

PET imaging was performed for $60 \mathrm{~min}$ for [18F]FDG and $30 \mathrm{~min}$ for [68Ga]FAPI (5-6mCi) after injection, with 5 bed positions of 3 min each. Emission PET data were acquired from the base of the skull to the upper thigh in 3D mode using a Discovery ST scanner (Discovery ${ }^{\mathrm{TM}} \mathrm{IQ}$; GE Healthcare), and then they were reconstructed with noncontrast CT (tube rotation time 1s/revolution, $120 \mathrm{kV}, 60 \mathrm{~mA}, 7.5 \mathrm{~mm} /$ rotation, scan length $867 \mathrm{~mm}$ ) by iterative reconstruction (ordered-subsets expectation maximization with 2 iterations and 30 subsets, field of view $=600 \mathrm{~mm}$, slice thickness $=3.27 \mathrm{~mm}$ ).

$\mathrm{PET}, \mathrm{CT}$, and fused whole-body images displayed in axial, coronal, and sagittal planes were available for review. A semi-quantitative analysis of tracer activity was measured as the maximal standardized uptake value (SUV $\mathrm{S}_{\max }$ ) of [18F]FDG or [68Ga]FAPI using the provided software (AW VolumeShare, GE Healthcare). [18F]FDG PET/CT and [68Ga]FAPI PET/CT images were evaluated both qualitatively and semi-quantitatively. For the semiquantitative analysis, polygonal regions of interest (ROIs) were first drawn on CT images and then copied to attenuation-corrected PET images using the Advantage Workstation (version 4.4, GE Healthcare). For tumors with a hypermetabolic lesion, ROIs were placed at every transaxial plane of CT images that contained the hypermetabolic lesion. Meanwhile, for those without visually discernible [18F]FDG or [68Ga]FAPI uptake, ROIs were drawn to cover the whole tumor. In cases of multiple malignant nodules, an ROI was drawn on the largest one. Maximum standardized uptake value $\left(\mathrm{SUV}_{\max }\right)$ was calculated with the injected dose and the patient's body weight. Any non-physiological uptake greater than background blood-pool activity or adjacent normal tissue background on [18F]FDG or [68Ga]FAPI PET was included in the study. Positive findings on PET were localized to anatomic images from the non-enhanced CT. The PET/CT findings were grouped as intramedullary bone lesions, lymph nodal metastasis, and distant metastasis. The lesion number and $\mathrm{SUV}_{\max }$ of the lesion with the highest pathological tracer accumulation were recorded for each bone lesion, lymph node, or distant metastasis site for both [18F]FDG and [68Ga]FAPI-04 PET/CT.

The PET/CT images were carefully evaluated by two experienced nuclear medicine physicians. Images were reviewed independently of the scans.

\subsection{Reference Standard}

Patients had undergone comprehensive re-evaluation, including clinical and hematological data, as well as the appraisal of bone lesions on an ultimate MRI. The histological specimen, hematological parameters (serum levels of beta-2-microglobulin $\left(\mathrm{B}_{2} \mathrm{M}\right)$, C-reactive protein (CRP) and lactate dehydrogenase (LDH), bone marrow aspirate (biopsy to determine infiltration by plasma cells), monoclonal proteins (M-proteins) in the serum or urine, serum-free light chain (FLC) ratio, and IgG isotype were also taken as the reference standard. Any performed therapeutic regimen wasregistered from the patients' medical record. Patients whose lesions were previously treated by external radiotherapy before $\mathrm{PET} / \mathrm{CT}$ imaging were excluded from this study to avoid confounding items.

\subsection{Statistical Analysis}

We performed descriptive analyses for the characteristics of patients. The BlandAltman analysis was used to evaluate the correlation between $\mathrm{SUV}_{\max }$ values of [68Ga]FAPI and [18F]FDG. Also, $p$-values of $<0.05$ were defined as statistically significant. All statistical analyses were performed using IBM SPSS Statistics Version 26 for Mac v15.41. 


\section{Results}

The study was conducted prospectively in 14 patients with MM. 50\% of the patients were female and 50\% were male. A comprehensive study was performed to demonstrate the following myeloma-related parameters in all eligible patients: serum M-protein; full immunoglobulin and free light chain type; serum levels of beta-2-microglobulin (B2M), $C$ reactive protein $(\mathrm{CRP})$ and lactate dehydrogenase $(\mathrm{LDH})$; bone marrow aspirate and biopsy to determine infiltration by plasma cells. Patients were staged according to the ISS and Durie Salmon PLUS staging system. The baseline characteristics of the patients were demonstrated in Table 1.

Table 1. Baseline characteristics of patients.

\begin{tabular}{|c|c|c|c|c|c|c|c|c|c|c|}
\hline No & A/G & $\begin{array}{c}\text { Plasma } \\
\text { Cell } \\
\text { Percentage }\end{array}$ & $\begin{array}{l}\text { Diagnostic } \\
\text { Localization }\end{array}$ & $\begin{array}{c}\text { Subtype of Ig (or } \\
\text { Non-Secretory Type) }\end{array}$ & $\begin{array}{c}\text { Type of } \\
\text { Light } \\
\text { Chain }\end{array}$ & ISS & LDH & CRP & $\begin{array}{c}\mathbf{B}_{2} \\
\text { Microglobulin }\end{array}$ & $\begin{array}{l}\text { Durie } \\
\text { Salmon } \\
\text { Staging }\end{array}$ \\
\hline 1 & $58 / \mathrm{m}$ & 80 & Bone marrow & $\operatorname{Ig} \mathrm{A}$ & Lambda & 1 & 215 & 6.5 & 2.8 & 3 \\
\hline 2 & $64 / f$ & 80 & Bone marrow & Non-secretory type & Lambda & 3 & 234 & 2.2 & 14.5 & 3 \\
\hline 3 & $39 / \mathrm{m}$ & 80 & Plasmocitoma & $\operatorname{IgG}$ & Kappa & 1 & 243 & 2.7 & 3.09 & 3 \\
\hline 4 & $65 / f$ & 70 & Bone marrow & $\operatorname{IgG}$ & Lambda & 3 & 157 & 2.5 & 14.3 & 2 \\
\hline 5 & $40 / f$ & 40 & Plasmocitoma & $\operatorname{IgG}$ & Kappa & 1 & 139 & 2.5 & 2.2 & 2 \\
\hline 6 & $58 / \mathrm{m}$ & 70 & Bone marrow & $\operatorname{IgG}$ & Lambda & 1 & 212 & 11.6 & 3.2 & 3 \\
\hline 7 & $81 / \mathrm{m}$ & 50 & Bone marrow & $\operatorname{IgG}$ & Kappa & 3 & 1366 & 23.7 & 9.2 & 3 \\
\hline 8 & $59 / f$ & 80 & Plasmocitoma & IgG & Kappa & 2 & 185 & 77.2 & 3.9 & 3 \\
\hline 9 & $54 / \mathrm{m}$ & 50 & Bone marrow & Non-secretory type & Lambda & 1 & 159 & 14 & 3.1 & 2 \\
\hline 10 & $55 / \mathrm{f}$ & 40 & Bone marrow & $\operatorname{Ig} \mathrm{A}$ & Lambda & 1 & 186 & 6.7 & 1.6 & 1 \\
\hline 11 & $57 / f$ & 40 & Plasmocitoma & IgG & Kappa & 2 & 146 & 1.6 & 2.7 & 3 \\
\hline 12 & $69 / f$ & 15 & Bone marrow & $\operatorname{IgG}$ & Lambda & 1 & 438 & 1.5 & 3 & 3 \\
\hline 13 & $58 / \mathrm{m}$ & 24 & Bone marrow & $\operatorname{IgG}$ & Lambda & 1 & 159 & 77 & 3.1 & 3 \\
\hline 14 & $66 / \mathrm{m}$ & 50 & Bone marrow & $\operatorname{Ig} A$ & Lambda & 3 & 125 & 1 & 8.3 & 3 \\
\hline
\end{tabular}

No: Patient's number; A/G: Age/Gender; m: Male, f: Female; LDH: Lactate dehydrogenase; CRP: C-reactive protein; B2M: Beta-2-microglobulin; ISS: International Staging System.

In 4 of 14 patients, [68Ga]FAPI PET/CT and [18F]FDG PET/CT did not detect any bone lesions. In 8 of the remaining 10 patients, [18F]FDG PET/CT detected bone lesions, but in this group, 6 patients showed a higher $S U V_{\max }$ value than [18F]FDG PET/CT in [68Ga]FAPI PET/CT. In contrast, 2 of 8 patients showed a higher $S_{\text {max }}$ value than [68Ga]FAPI PET/CT in [18F]FDG PET/CT. Moreover, [68Ga]FAPI PET/CT detected bone lesions in two patients which werenot detected by [18F]FDG PET/CT. Also, in five patients, [68Ga]FAPI PET/CT showed more bone lesions in comparison with [18F]FDG PET/CT. Only in one patient [18F]FDG PET/CT show more bone lesions (Table 2).

Table 2. Number and $\mathrm{SUV}_{\max }$ values of lesions detected by [18F]FDG PET/CT and [68Ga]FAPI $\mathrm{PET} / \mathrm{CT}$ in bone and extramedullary involvement.

\begin{tabular}{|c|c|c|c|c|c|c|}
\hline No & $\begin{array}{l}\text { Number of Bone } \\
\text { Lesions with FDG }\end{array}$ & $\begin{array}{l}\text { FDG SUV }_{\max } \\
\text { Value }\end{array}$ & $\begin{array}{l}\text { Number of Bone } \\
\text { Lesions with FAPI }\end{array}$ & $\begin{array}{l}\text { FAPI SUV }_{\max } \\
\text { Value }\end{array}$ & $\begin{array}{l}\text { Extramedullary } \\
\text { Involvement }^{\text {a }}\end{array}$ & $\begin{array}{l}\text { Extramedullary } \\
\text { Involvement }{ }^{b}\end{array}$ \\
\hline 1 & 1 & 4.1 & 10 & 13.6 & $8.1^{1}$ & $13^{1}$ \\
\hline 2 & 0 & 0 & 6 & 6.5 & & \\
\hline 3 & 4 & 5.3 & 13 & 6.6 & $2.7^{2}$ & $14.7^{2}$ \\
\hline 4 & 0 & 0 & 0 & 0 & & \\
\hline 5 & 2 & 2.1 & 2 & 8.4 & & \\
\hline 6 & 0 & 0 & 8 & 13.1 & & \\
\hline 7 & 0 & 0 & 0 & 0 & & \\
\hline 8 & 6 & 5.6 & 7 & 4.6 & $11.7^{3}$ & $5.7^{3}$ \\
\hline 9 & 3 & 15.4 & 3 & 13.6 & & \\
\hline 10 & 0 & 0 & 0 & 0 & & \\
\hline 11 & 14 & 6.6 & 4 & 8.1 & & \\
\hline 12 & 0 & 0 & 0 & 0 & & \\
\hline 13 & 6 & 5.5 & 9 & 8.2 & & \\
\hline 14 & 2 & 4.6 & 4 & 10.3 & & \\
\hline
\end{tabular}

\footnotetext{
${ }^{a}$ Metastatic region and FDG SUV $\max .{ }^{b}$ Metastatic region and FAPI SUV $\max .{ }^{1}$ Lung. ${ }^{2}$ Presacral lymph node.
}

${ }^{3}$ Soft tissue mass in the lateral neighborhood of the right maxillary sinus. 
Mean \pm standard deviation (SD) of $\mathrm{SUV}_{\max }$ values of bone lesions in [18F]FDG and [68Ga]FAPI were $6.15 \pm 3.97(n=8)$ and $9.30 \pm 3.22(n=10)$, respectively. There was no statistically significant difference between these imaging modalities in terms of $\mathrm{SUV}_{\max }$ values of bone lesions ( $p=0.081)$.

Both PET/CT imaging modalities demonstrated extramedullary involvement in the following locations in three patients: lung, presacral lymph node, and soft tissue mass lateral to the right maxillary sinus. Among these involvements, higher $\mathrm{SUV}_{\max }$ values were observed in the lung (Figure 1B) and presacral lymph node (Figure 2B) with [68Ga]FAPI compared to [18F]FDG. However, the soft tissue mass (indicated in Table 1) showed a

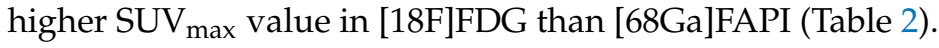
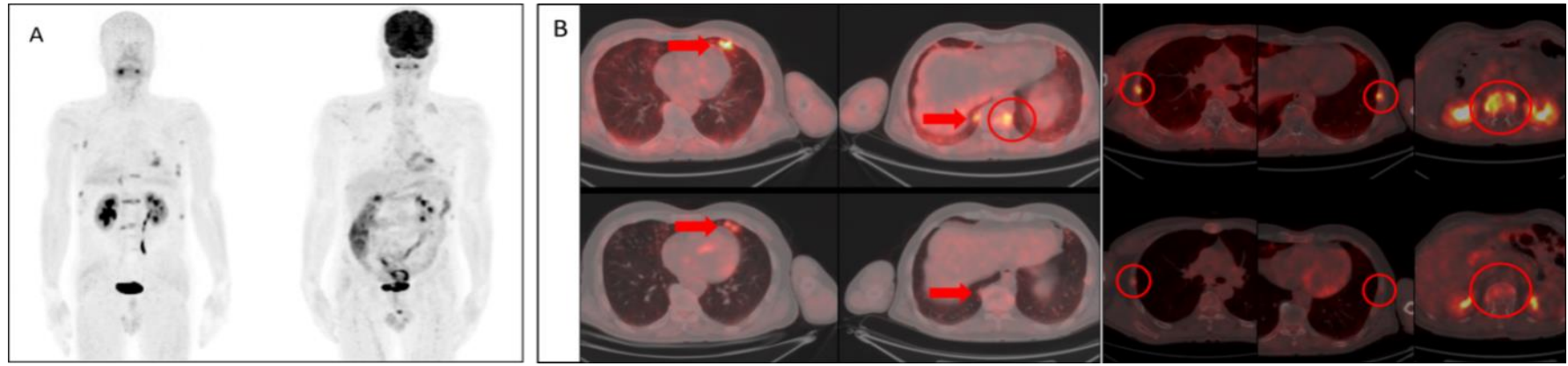

Figure 1. Patient No 1. 58 year-old-male diagnosed with MM. (A) Visual comparison of maximum intensity projections of both modalities (Left [68Ga]FAPI, right [18F]FDG) showed superior image quality and higher specific activity retentions with [68Ga]FAPI PET/CT. (B) In axial plane evaluations, both imaging methods demonstrated extramedullary involvement of MM in the lungs (Arrows); however, $\mathrm{SUV}_{\max }$ values of the lesions were noted to be higher in [68Ga]FAPI PET/CT (Upper row). Also, significantly higher activity uptake was observed in the bone lesions (Circles) with [68Ga]FAPI (Upper row) when compared to [18F]FDG (Lower row).
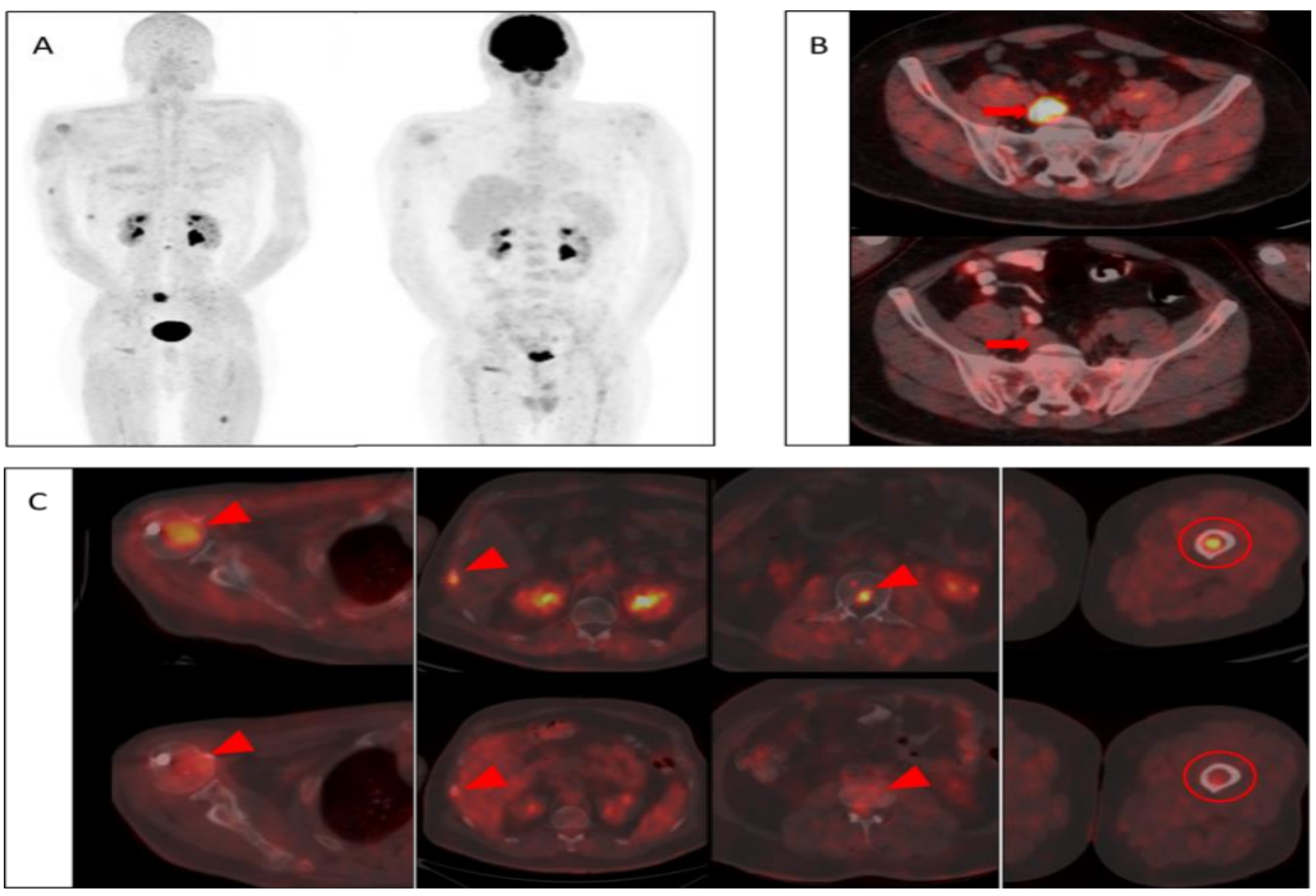

Figure 2. Patient No 3. 39-year-old male diagnosed with MM. (A) Visual comparison of maximum intensity projections of both modalities showed superior image quality and higher specific activity 
retentions in [68Ga]FAPI PET/CT (Left) without non-specific diffuse medullary uptake as in [18F]FDG PET/CT (Right). (B) In axial plane evaluations, both imaging methods demonstrated extramedullary involvement of MM in the presacral lymph node (Arrows); however, $\mathrm{SUV}_{\max }$ value of the lymph node were noted to be significantly higher in [68Ga]FAPI PET/CT (Upper row). (C) In the visual evaluation of the bone lesions (Arrow heads), [68Ga]FAPI PET/CT (Upper row) demonstrated higher activity retentions in all locations with significantly lower background activity compared to [18F]FDG PET/CT (Lower row). Also, more pronounced focal activity retention was observed in the intramedullary region of the left femur with [68Ga]FAPI PET/CT (Upper row) compared to [18F]FDG PET/CT (Lower row), without morphological pathology (Circles).

\section{Discussion}

Imaging modalities in MM play an important role in diagnosis, determining disease dissemination, and evaluation of response to treatment. On whole-body x-radiography (WBXR), multiple myeloma lesions typically have a perforated osteolytic appearance. However, for it to be detected as a lytic lesion on radiography, at least $50 \%$ of the relevant trabecular bone must be destroyed. On the other hand, computed tomography (CT) has been found to be more sensitive than WBXR in detecting lytic lesions with less than 5\% trabecular bone destruction $[5,20,21]$. Both WBXR and CT assess the damage of tumor cells to mineralized bone tissue, but do not provide information on tumor cell viability or activity. On the other hand, magnetic resonance imaging (MRI) and PET/CT are more functional imaging methods, as they can evaluate the diffusion of interstitial water molecules and glucose uptake, which are markers of viability in tumor tissue [22].The International Myeloma Working Group has recommended that use of [18F]FDG PET/CT in MM could provide beneficial information about the metabolism of plasma cells. The most important advantage of [18F]FDG PET/CT is its ability to assess disease burden with highaccuracy and to distinguish metabolic activity among various lesions [23,26]. However, utilization of [18F]FDG PET/CT in patients with MM has some limitations. Studies have shown that false positive results can be detected with [18F]FDG PET/CT in certain situations, such as low [18F]FDG affinity, recent steroid treatment, hyperglycemia, non-specific bone marrow involvements, and small lytic lesions in the skull close to the brain [10,11]. High [18F]FDG affinity associated with poor prognosis and possible false negative results may have a negative clinical impact on the initial assessment of MM [12]. Therefore, novel imaging modalities, such as [68Ga]FAPI PET/CT, may be useful.

In the current study, [68Ga]FAPI PET/CT showed high quality images with specific tumor activity. Also, there were no non-specific involvements as well as favorable low background activity in visual evaluations in agreement with the literature $[18,19,27-29]$. In two of the patients, [68Ga]FAPI PET/CT showed multiple bone lesions with considerably higher SUV max values, while [18F]FDG PET/CT have not shown hyper-metabolic lesions. Also, more bone lesions were detected in five of the patients with [68Ga]FAPI PET/CT. In six of the patients, both modalities showed similar results, and in one of the patients, more foci were identified in [18F]FDG PET/CT when compared to [68Ga]FAPI PET/CT. Also in a patient, it is noteworthy that [68Ga]FAPI PET/CT showed focal intramedullary bone involvement without morphological pathology, whereas [18F]FDG PET/CT showed no significant uptake (Figure 2C). However, there was no statistically significant difference between these imaging modalities in terms of $\mathrm{SUV}_{\max }$ values of bone lesions (Figure 3). 


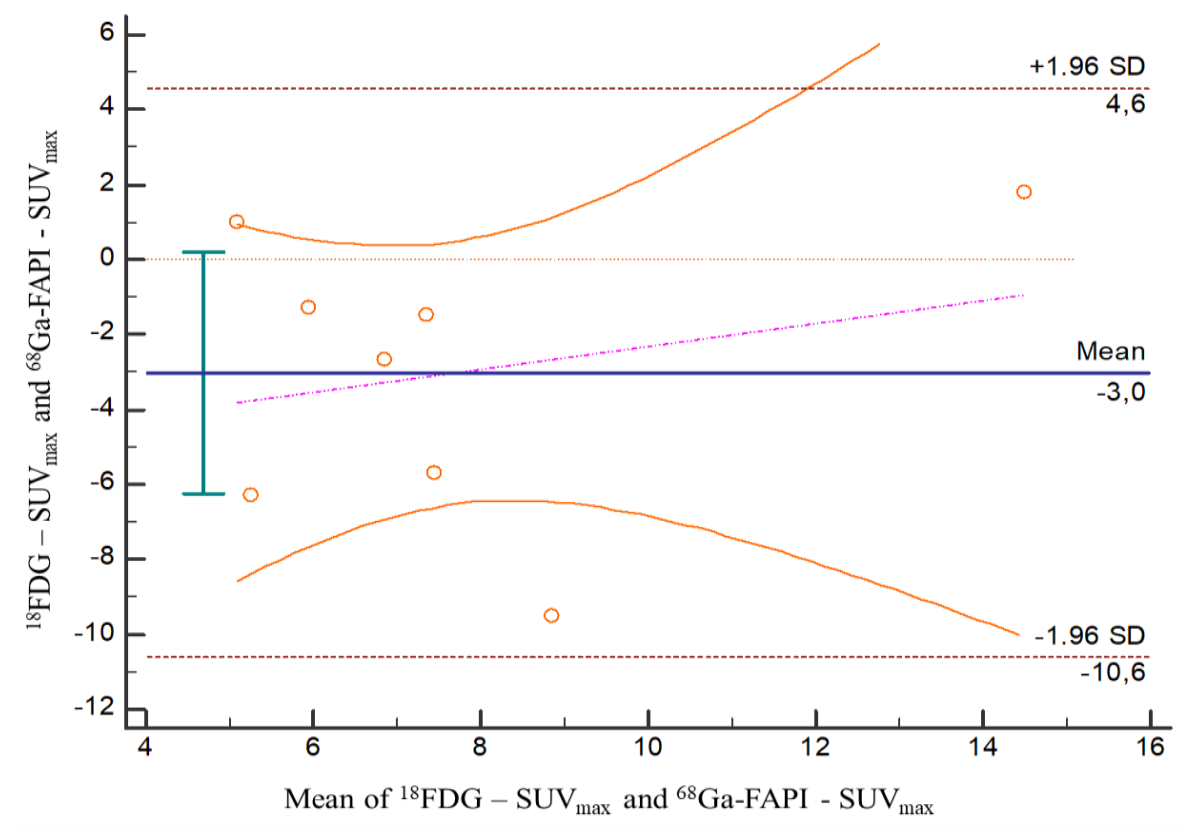

Figure 3. Bland-Altman plot for comparison between $\mathrm{SUV}_{\max }$ values of bone lesions detected in

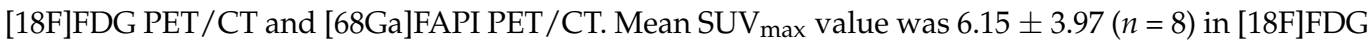
and $9.30 \pm 3.22(n=10)$ in [68Ga]FAPI. There was no statistically significant difference between $\mathrm{SUV}_{\max }$ values of bone lesions detected in both modalities $(p=0.081)$.

Both imaging methods showed extramedullary involvement in three patients with different activity characteristics. Extramedullary involvement of two patients showed higher [68Ga]FAPI uptake than [18F]FDG, however more pronounced [18F]FDG retention was observed in the other patient.

In a recent study, lesion-based comparison of [68Ga]FAPI PET/CT and [18F]FDG PET/CT in three patients with MM demonstrated that [18F]FDG had greater sensitivity than [68Ga]FAPI. Furthermore, [18F]FDG PET/CT showed significantly higher $\mathrm{SUV}_{\max }$ values compared to [68Ga]FAPI PET/CT [29]. In contrast, no statistically significant association was found between the SUV $\max$ values of both imaging methods in the current study.

In addition to the relatively small sample size, our study may have limitations as it did not include histopathological confirmation of all metastatic lesions, except primary lesions and target lesions.

\section{Conclusions}

In conclusion, no significant superiority was observed in [68Ga]FAPI PET/CT over [18F]FDG PET/CT in patients with MM in this study. The results of our study did not make a significant contribution to changing treatment management in the patients. However, [68Ga]FAPI PET/CT can be utilized as a complementary imaging method to [18F]FDG $\mathrm{PET} / \mathrm{CT}$ in some settings, especially in low [18F]FDG affinity and inconclusive cases. Considering the favorable aspects of [68Ga]FAPI PET/CT in MM, such as low background activity, absence of non-specific bone marrow, and physiological brain involvement, further studies with a larger sample size should be conducted.

Author Contributions: Conceptualization: U.E. and E.S.; methodology: I.D.; software: B.K.; validation: E.S. and Y.Z.C.; formal analysis: I.D.; investigation: T.K. and D.M.A.; data curation: I.D.; writing-original draft preparation: H.H.S., M.O. and Y.B.C.; writing-review and editing: G.A., Y.B.C. and M.O.; visualization: U.C. and V.M.; supervision: U.E.; project administration: U.E. All authors have read and agreed to the published version of the manuscript.

Funding: This research received no external funding. 
Institutional Review Board Statement: All procedures performed in studies involving human participants were in accordance with the ethical standards of the institutional and/or national research committee and with the 1964 Helsinki declaration and its later amendments or comparable ethical standards. Ethic Committee Name: Gaziantep University Clinical Research Ethics Committee. Approval Code: 304. Approval Date: 15 September 2021.

Informed Consent Statement: Informed consent was obtained from all subjects involved in the study.

Data Availability Statement: All data generated or analyzed during this study are included in this article [and/or] its supplementary material files. Further enquiries can be directed to the corresponding author.

Conflicts of Interest: The authors declare no conflict of interest.

\section{References}

1. Terpos, E.; Dimopoulos, M.-A. Myeloma bone disease: Pathophysiology and management. Ann. Oncol. 2005, $16,1223-1231$. [CrossRef] [PubMed]

2. Zamagni, E.; Tacchetti, P.; Cavo, M. Imaging in multiple myeloma: How? When? Blood 2019, 133, 644-651. [CrossRef] [PubMed]

3. Kyle, R.A.; Rajkumar, S.V. Multiple myeloma. N. Engl. J. Med. 2004, 351, 1860-1873. [CrossRef] [PubMed]

4. Roodman, G.D. Pathogenesis of myeloma bone disease. Leukemia 2009, 23, 435-441. [CrossRef]

5. Hanrahan, C.J.; Carl, R.C.; Julia, R.C. Current concepts in the evaluation of multiple myeloma with MR imaging and FDG PET/CT 1. Radiographics 2010, 30, 127-142. [CrossRef]

6. Hughes, N.M.; Jacene, H.A. PET Imaging for Hematologic Malignancies. Radiol. Clin. N. Am. 2021, 59, 705-723. [CrossRef]

7. Schirrmeister, H.; Bommer, M.; Buck, A.; Müller, S.; Messer, P.; Bunjes, D.; Döhner, H.; Bergmann, L.; Reske, S.N. Initial results in the assessment of multiple myeloma using F-18 FDG PET. Eur. J. Nucl. Med. Mol. Imaging 2002, 29, 361-366. [CrossRef]

8. Zamagni, E.; Nanni, C.; Patriarca, F.; Englaro, E.; Castellucci, P.; Geatti, O.; Tosi, P.; Tacchetti, P.; Cangini, D.; Perrone, G.; et al. A prospective comparison of 18F-fluorodeoxyglucose positron emission tomography-computed tomography, magnetic resonance imaging and whole-body planar radiographs in the assessment of bone disease in newly diagnosed multiple myeloma. Haematologica 2007, 92, 50-55. [CrossRef]

9. Beyer, R.J., 3rd; Mulligan, M.E.; Smith, S.E.; Line, B.R.; Badros, A.Z. Comparison of imaging with FDG PET/CT with other imaging modalities in myeloma. Skeletal Radiol. 2006, 35, 632-640. [CrossRef]

10. Rasche, L.; Angtuaco, E.; McDonald, J.E.; Buros, A.; Stein, C.; Pawlyn, C.; Thanendrarajan, S.; Schinke, C.; Samant, R.; Yaccoby, S.; et al. Low expression of hexokinase-2 is associated with false-negative FDG-positron emission tomography in multiple myeloma. Blood 2017, 130, 30-34. [CrossRef]

11. Jung, S.-H.; Kwon, S.Y.; Min, J.-J.; Bom, H.-S.; Ahn, S.-Y.; Jung, S.-Y.; Lee, S.-S.; Park, M.-R.; Yang, D.-H.; Ahn, J.-S.; et al. 18F-FDG PET / CT is useful for determining survival outcomes of patients with multiple myeloma classified as stage II and III with the revised International Staging System. Eur. J. Nucl. Med. Mol. Imaging 2019, 46, 107-115. [CrossRef] [PubMed]

12. Zhong, X.; Diao, W.; Zhao, C.; Jia, Z. Fluorodeoxyglucose-avid focal lesions and extramedullary disease on 18F-FDG $\mathrm{PET} /$ computed tomography predict the outcomes of newly diagnosed symptomatic multiple myeloma patients. Nucl. Med. Commun. 2020, 41, 950-958. [CrossRef] [PubMed]

13. Scanlan, M.J.; Raj, B.K.; Calvo, B.; Garin-Chesa, P.; Sanz-Moncasi, M.P.; Healey, J.; Old, L.J.; Rettig, W.J. Molecular cloning of fibroblast activation protein alpha, a member of the serine protease family selectively expressed in stromal fibroblasts of epithelial cancers. Proc. Natl. Acad. Sci. USA 1994, 91, 5657-5661. [CrossRef] [PubMed]

14. Simková, A.; Busek, P.; Sedo, A.; Konvalinka, J. Molecular recognition of fibroblast activation protein for diagnostic and therapeutic applications. Biochim. Biophys. Acta (BBA)-Proteins Proteom. 2020, 1868, 140409. [CrossRef] [PubMed]

15. Chen, X.; Song, E. Turning foes to friends: Targeting cancer-associated fibroblasts. Nat. Rev. Drug Discov. 2019, 18, 99-115. [CrossRef]

16. Loktev, A.; Lindner, T.; Burger, E.M.; Altmann, A.; Giesel, F.; Kratochwil, C.; Debus, J.; Marmé, F.; Jäger, D.; Mier, W.; et al. Development of novel FAP-targeted radiotracers with improved tumor retention. J. Nucl. Med. 2019, 60, 1421-1429. [CrossRef]

17. Lindner, T.; Loktev, A.; Altmann, A.; Giesel, F.; Kratochwil, C.; Debus, J.; Jäger, D.; Mier, W.; Haberkorn, U. Development of quinoline-based theranostic ligands for the targeting of fibroblast activation protein. J. Nucl. Med. 2018, 59, 1415-1422. [CrossRef]

18. Giesel, F.L.; Kratochwil, C.; Lindner, T.; Marschalek, M.M.; Loktev, A.; Lehnert, W.; Debus, J.; Jäger, D.; Flechsig, P.; Altmann, A.; et al. 68Ga-FAPI PET/CT: Biodistribution and preliminary dosimetry estimate of 2 DOTA-containing FAPI targeting agents in patients with various cancers. J. Nucl. Med. 2019, 60, 386-392. [CrossRef]

19. Kratochwil, C.; Flechsig, P.; Lindner, T.; Abderrahim, L.; Altmann, A.; Mier, W.; Adeberg, S.; Rathke, H.; Röhrich, M.; Winter, H.; et al. 8Ga-FAPI PET/CT: Tracer uptake in 28 different kinds of cancer. J. Nucl. Med. 2019, 60, 801-805. [CrossRef]

20. Angtuaco, E.J.C.; Fassas, A.B.T.; Walker, R.; Sethi, R.; Barlogie, B. Multiple myeloma: Clinical review and diagnostic imaging. Radiology 2004, 231, 11-23. [CrossRef]

21. Amos, B.; Agarwal, A.; Kanekar, S. Imaging of Multiple Myeloma. Hematol. Clin. N. Am. 2016, 30, 843-865. [CrossRef] [PubMed] 
22. Landgren, O.; Kyle, R.A.; Pfeiffer, R.M.; Katzmann, J.A.; Caporaso, N.E.; Hayes, R.B.; Dispenzieri, A.; Kumar, S.; Clark, R.J.; Baris, D.; et al. Monoclonal gammopathy of undetermined significance (MGUS) consistently precedes multiple myeloma: A prospective study. Blood 2009, 113, 5412-5417. [CrossRef] [PubMed]

23. Weiss, B.M.; Abadie, J.; Verma, P.; Howard, R.S.; Kuehl, W.M. A monoclonal gammopathy precedes multiple myeloma in most patients. Blood 2009, 113, 5418-5422. [CrossRef] [PubMed]

24. Kyle, R.A.; Remstein, E.D.; Therneau, T.M.; Dispenzieri, A.; Kurtin, P.J.; Hodnefield, J.M.; Larson, D.R.; Plevak, M.F.; Jelinek, D.F.; Fonseca, R.; et al. Clinical course and prognosis of smoldering (asymptomatic) multiple myeloma. N. Engl. J. Med. 2007, 356, 2582-2590. [CrossRef]

25. Rajkumar, S.V. Updated Diagnostic Criteria and Staging System for Multiple Myeloma. Am. Soc. Clin. Oncol. Educ. Book 2016, 35, e418-e423. [CrossRef]

26. Cavo, M.; Terpos, E.; Nanni, C.; Moreau, P.; Lentzsch, S.; Zweegman, S.; Hillengass, J.; Engelhardt, M.; Usmani, S.Z.; Vesole, D.H.; et al. Role of 18F-FDG PET/CT in the diagnosis and management of multiple myeloma and other plasma cell disorders: A consensus statement by the International Myeloma Working Group. Lancet Oncol. 2017, 18, e206-e217. [CrossRef]

27. Chen, H.; Pang, Y.; Wu, J.; Zhao, L.; Hao, B.; Wu, J.; Wei, J.; Wu, S.; Zhao, L.; Luo, Z.; et al. Comparison of [68Ga]Ga-DOTA-FAPI-04 and [18F] FDG PET/CT for the diagnosis of primary and metastatic lesions in patients with various types of cancer. Eur. J. Nucl. Med. Mol. Imaging 2020, 47, 1820-1832. [CrossRef]

28. Chen, H.; Zhao, L.; Ruan, D.; Pang, Y.; Hao, B.; Dai, Y.; Wu, X.; Guo, W.; Fan, C.; Wu, J.; et al. Usefulness of [68Ga]Ga-DOTA-FAPI$04 \mathrm{PET} / \mathrm{CT}$ in patients presenting with inconclusive [18F]FDG PET/CT findings. Eur. J. Nucl. Med. Mol. Imaging 2021, $48,73-86$. [CrossRef]

29. Lan, L.; Liu, H.; Wang, Y.; Deng, J.; Peng, D.; Feng, Y.; Wang, L.; Chen, Y.; Qin, L. The potential utility of [68Ga]Ga-DOTA-FAPI-04 as a novel broad-spectrum oncological and non-oncological imaging agent-comparison with [18F]FDG. Eur. J. Nucl. Med. Mol. Imaging 2022, 49, 963-979. [CrossRef] 